\title{
Excessive levels of nitric oxide in rat model of Parkinson's disease induced by rotenone
}

\author{
ZHONG-KUI XIONG ${ }^{1,2,3^{*}}$, JUAN LANG ${ }^{4 *}$, GANG XU ${ }^{5 *}$, HAI-YU LI ${ }^{6}$, \\ YUN ZHANG $^{7}$, LEI WANG ${ }^{3}$, YAO SU ${ }^{3}$ and AI-JING SUN ${ }^{8 *}$ \\ ${ }^{1}$ Department of Radiotherapy, Shaoxing Second Hospital, Shaoxing, Zhejiang 312000; ${ }^{2}$ Department of Radiotherapy, \\ Shaoxing Campus, The First Affiliated Hospital, School of Medicine, Zhejiang University, Shaoxing, Zhejiang 312000; \\ ${ }^{3}$ Department of Clinical Medicine, Shaoxing University School of Medicine, Shaoxing, Zhejiang 312099; \\ ${ }^{4}$ Medical Research Center, Shaoxing People's Hospital, Zhejiang University, Shaoxing, Zhejiang 312000; \\ ${ }^{5}$ Department of Radiotherapy, Jiangsu University Affiliated People's Hospital, Zhenjiang, Jiangsu 212002; \\ Departments of ${ }^{6}$ Laboratory Medicine and ${ }^{7}$ Basic Medicine, Shaoxing University School of Medicine, \\ Shaoxing, Zhejiang 312099; ${ }^{8}$ Department of Pathology, Shaoxing People's Hospital, Zhejiang University, \\ Shaoxing, Zhejiang, Shaoxing, Zhejiang 312000, P.R. China
}

Received March 26, 2014; Accepted October 28, 2014

DOI: $10.3892 /$ etm.2014.2099

\begin{abstract}
Systemic rotenone models of Parkinson's disease (PD) are highly reproducible and may provide evidence on the pathogenesis of PD. In the present study, male Sprague-Dawley rats (1-year-old) were subcutaneously administered with rotenone $(1.5 \mathrm{mg} / \mathrm{kg} /$ day) for six days and observed for the following three weeks. Compared with the control rats, a significant decrease was observed in the body weight and a marked increase was observed in the areas under the behavioral scoring curves in the rotenone-treated rats. Immunohistochemical staining revealed that the abundance of nigral tyrosine hydroxylase (TH)-positive neurons was markedly reduced following rotenone treatment. ELISA and neurochemical assays demonstrated a significant increase in the levels of nitric oxide (NO) and NO synthase, whereas a marked decrease was observed in the thiol levels in the brains of the rotenone-treated rats. Thus, subacute rotenone treatment was found to induce behavioral deficits and the loss of nigral TH-positive neurons which may be associated with the excessive levels of NO in the rat brains.
\end{abstract}

Correspondence to: Dr Ai-Jing Sun, Department of Pathology, Shaoxing People's Hospital, Zhejiang University, 568 North Zhongxing Road, Shaoxing, Zhejiang 312000, P.R. China

E-mail: sun_aijing@hotmail.co.jp

Dr Zhong-Kui Xiong, Department of Radiotherapy, Shaoxing Campus, The First Affiliated Hospital, School of Medicine, Zhejiang University, 123 West Yan'an Road, Shaoxing, Zhejiang 312000, P.R. China

E-mail: xiongzk@yeah.net

*Contributed equally

Key words: nitric oxide, rotenone, Parkinson's disease

\section{Introduction}

Parkinson's disease (PD) is a common neurodegenerative disease, characterized by the selective degeneration and loss of nigral dopaminergic neurons leading to striatal dopamine (DA) depletion $(1,2)$. Typical symptoms include the loss of $\sim 50 \%$ of dopaminergic neurons in the substantia nigra and $>70-80 \%$ loss of DA in the striatum $(1,3,4)$. PD is mainly regarded as a disease of aging (5), affecting 1-2\% of the world's population aged $\geq 60$ years and almost $4 \%$ of individuals aged $>85$ years $(6,7)$.

Increasing evidence has indicated that excessive nitric oxide (NO) production contributes to the aging and PD development processes $(8,9)$. Aging has been shown to alter the brain arginine metabolism of male Sprague-Dawley (SD) rats (10). In addition, a previous study has demonstrated that the mRNA and protein expression of neuronal NO synthase (NOS) is age-dependent in the brain cortex of rats (11). Higher levels of neuronal NOS (nNOS) and inducible NOS (iNOS) were observed in the substantia nigra of PD patients and animal models $(12,13)$. Furthermore, genes coding for NOS have been shown to generate excess NO, contributing to neurodegeneration in PD (12). The iNOS expression increase has been shown to be inversely correlated with the immunolabeling of tyrosine hydroxylase (TH), a marker of DA neurons. A previous study has demonstrated that animals pretreated with the NOS inhibitor, N(G)-nitro-L-arginine methyl ester, exhibited complete protection against amphetamine-induced body rotations (14).

Epidemiological evidence has indicated that pesticides and other environmental exposures may play a role in the development of idiopathic PD $(15,16)$. Rotenone is a natural, plant-derived pesticide. A previous study demonstrated that exposure to rotenone was associated with an increased risk of PD development in the study population (17). In addition, NOS genes may interact with each other or with 
environmental factors in PD (12). Chronic rotenone administration has been shown to lead to significant injury of the nigrostriatal system, which is mediated by increased NO generation $(18,19)$. Compared with young rats, middle-aged rats (12-14 months old) have been shown to be more sensitive to rotenone (20). However, the levels of NO in the brain tissue of middle-aged rats that were subacutely induced by rotenone have been rarely reported.

In the present study, a subacute rotenone-induced rat model was established using middle-aged SD rats to investigate the effect of rotenone on NO production in the brain.

\section{Materials and methods}

Animals and rotenone treatment. All the experimental procedures were approved by the Animal Care and Use Committee of Shaoxing University School of Medicine (Shaoxing, China). Male SD rats ( $\mathrm{n}=25$; 1-year-old) were purchased from the Zhejiang Provincial Experimental Animal Center (Hangzhou, China). The rats were pair-housed in an environmentally controlled facility $\left(12 / 12 \mathrm{~h}\right.$ light/dark cycle; temperature, $22 \pm 2^{\circ} \mathrm{C}$; relative humidity, $50 \pm 5 \%$ ) and were provided with food and water ad libitum. The rats were randomly divided into two groups, including the control $(n=9)$ and rotenone-treated $(n=16)$ groups.

Prior to the experimental procedure, the rats were acclimatized for one week. Rotenone (Sigma-Aldrich, St. Louis, MO, USA) was dissolved in dimethyl sulfoxide to prepare a solution with concentration of $50 \mathrm{mg} / \mathrm{ml}$, which was further diluted to $2 \mathrm{mg} / \mathrm{ml}$ using rapeseed oil. Rats in the rotenone-treated group were subcutaneously injected with $1.5 \mathrm{mg} / \mathrm{kg} /$ day rotenone solution for six days. Rats in the control group were injected with the same solution dose, without rotenone.

Behavioral study. The behavioral scoring method described by Chen et al was used to daily assess behavioral alterations in the rats between the day prior to rotenone treatment and three weeks after termination of the treatment (total 27 days) (21). The rat behavioral scoring was as follows: 0 , no symptoms; 1 , dirty and bristling fur, bending of the back, attenuation of resisting arrest or decrease in passive movement; 2, significant decrease in passive movement, bradykinesia, tremors or unsteady movement; 4, inability to move in a straight line or unilateral rotation; 6 , unilateral lying posture or paralysis, walking or eating difficulties; 8, complete unilateral paralysis, spasms, marked weight loss or inability to eat; 10 , near-mortality or mortality.

TH immunohistochemical examination. After three weeks of observation following treatment completion, the rats were intraperitoneally anesthetized with pentobarbital sodium $(50 \mathrm{mg} / \mathrm{kg}$ body weight) and sacrificed by cervical dislocation. The rats were intracardially perfused with $30 \mathrm{ml} 0.01 \mathrm{M}$ phosphate-buffered saline (PBS; pH 7.4), followed by $200 \mathrm{ml} 4 \%$ formaldehyde in PBS. The rat brains were dissected and postfixed in $4 \%$ formaldehyde in PBS at room temperature for $2 \mathrm{~h}$. Next, the brains were cryoprotected in $20 \%$ sucrose in PBS at $4^{\circ} \mathrm{C}$ overnight, embedded in paraffin and cut into $25-\mu \mathrm{m}$ coronal sections using a microtome (Leica Microsystems Nussloch GmbH, Nussloch, Germany). Prior to staining, the sections were dewaxed by washing three times with xylene for $15 \mathrm{~min}$ and infused in 100, 95 and $75 \%$ alcohol, followed by water ( 5 min each time).
The sections were treated with $0.1 \%$ Triton X-100 (Aladdin Industrial Corporation, Shanghai, China) and $3.0 \% \mathrm{H}_{2} \mathrm{O}_{2}$ in $0.01 \mathrm{M}$ PBS ( $\mathrm{pH}$ 7.4) for $10 \mathrm{~min}$. Subsequently, the sections were treated with $3 \%$ bovine serum albumin (Amresco, Solon, $\mathrm{OH}, \mathrm{USA}$ ) in PBS for $15 \mathrm{~min}$ to reduce nonspecific antibody binding. Next, the samples were incubated with rabbit anti-TH polyclonal antibody (1:100; Millipore, Billerica, MA, USA) in PBS containing $3 \%$ bovine serum albumin at $4^{\circ} \mathrm{C}$ overnight and $37^{\circ} \mathrm{C}$ for $30 \mathrm{~min}$. The sections were washed with PBS and incubated with biotinylated sheep anti-rabbit secondary immunoglobylin G (1:10; EarthOx, LLC, San Francisco, CA, USA) for $1 \mathrm{~h}$ at room temperature, followed by incubation with streptavidin-biotin complex (1:10; Boster Bio-Engineering, Co., Ltd., Wuhan, China) in PBS (pH 7.4) for 30 min. Next, immunolabeling was visualized using 0.05\% 3,3'-diaminobenzidine (Boster Bio-Engineering, Co., Ltd.) $/ 0.005 \% \mathrm{H}_{2} \mathrm{O}_{2}$ in Tris-buffered saline and rinsed in water to terminate the reaction. Finally, the sections were dehydrated, cleared and sealed for image capture. Images were captured with an Eclipse TS100 microscope (Nikon Corporation, Tokyo, Japan)

Preparation of brain homogenate supernatant. Half of the brain tissue from each rat, with the exception of the cerebellum, was weighed and homogenized in 9 volumes of normal saline in an ice bath for $8 \mathrm{~min}$ ( $3 \mathrm{sec}$ on and $5 \mathrm{sec}$ off) using a JY92-IIDN ultrasonic cell crusher (Ningbo Scientz Biotechnology Co., Ltd., Ningbo, China). The samples were subsequently centrifuged at $2,556 \mathrm{x}$ g for $10 \mathrm{~min}$ at $4^{\circ} \mathrm{C}$. The total concentration of supernatant proteins was determined using a BCA Protein Assay kit (Pierce Biotechnology, Inc., Rockford, IL, USA). The supernatants were stored at $-80^{\circ} \mathrm{C}$ for subsequent ELISA and neurochemical analyses.

ELISA analysis. ELISA kits (RapidBio, West Hills, CA, USA) were used to determine the levels of NOS and superoxide radicals in the supernatants of the rat brain homogenates, according to the manufacturer's instructions. A total of $50 \mu \mathrm{l}$ samples or reference standards were pipetted into the microplate wells and incubated for $10 \mathrm{~min}$ at room temperature. An enzyme-linked antibody (EarthOx Life Sciences, Millbrae, CA, USA) specific to NOS or superoxide radicals was added to the wells and incubated for a further $2 \mathrm{~h}$ in order to bind to NOS or superoxide radical ligand. A substrate solution consisting of $\mathrm{H}_{2} \mathrm{O}_{2}$ and tetramethyl benzidine was added to the samples and the intensity at $450 \mathrm{~nm}$ was measured using an automicroplate reader (Model 2010; Anthos Labtec Instruments, GmbH, Salzburg, Austria). A YJZXM008 autowasher (Anthos Labtec Instruments, GmbH) was used to wash the microplate wells. Each sample was assessed in duplicate.

Neurochemical analysis. The activity of superoxide dismutase (SOD) in the supernatants of brain homogenates was determined using a colorimetric total mercapto(-SH) measurement kit. assay kit (Nanjing Jiancheng Bioengineering Institute, Nanjing, China), according to the manufacturer's instructions. Briefly, the supernatants and reagents were mixed and then incubated at $37^{\circ} \mathrm{C}$ for $40 \mathrm{~min}$, followed by $10 \mathrm{~min}$ of chromogenic reaction. SOD activity was determined using a spectrophotometric method (22) to measure the absorbance at $550 \mathrm{~nm}$. The results are expressed as units of SOD activity units/mg protein, where 

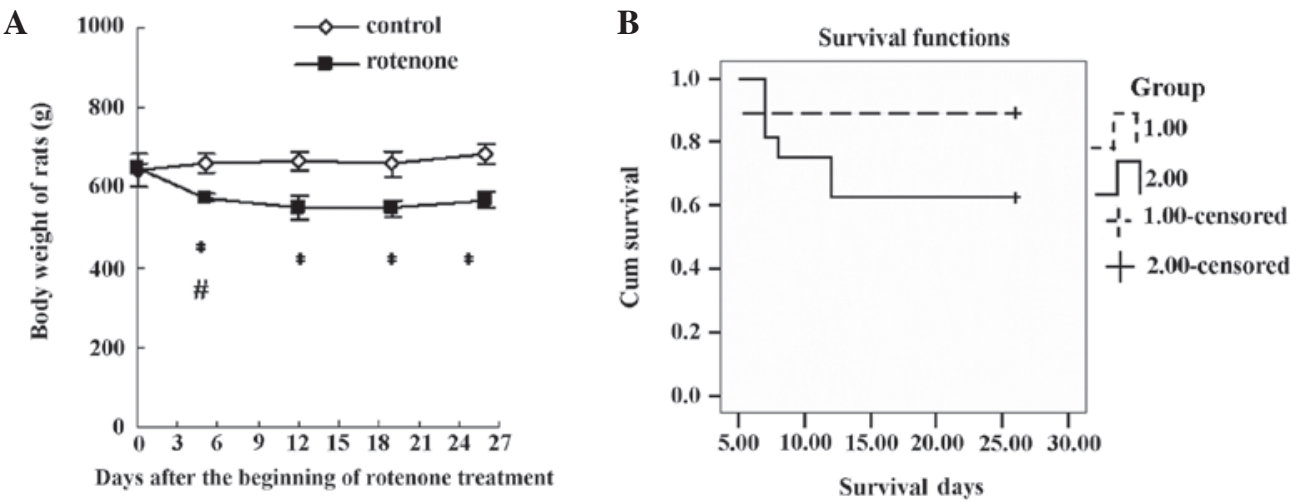

Figure 1. (A) Effect of rotenone on the body weight of rats prior to and at weeks $0,1,2$ and 3 following rotenone treatment (mean \pm standard error of the mean). (B) Survival rates of the rotenone-treated and control rats. The control and rotenone-treated groups consisted of 9 and 16 rats, respectively, at the beginning of the experimental procedure and 8 and 10 rats, respectively, at the end of the experimental procedure. ${ }^{*} \mathrm{P}<0.05$, vs. control group; ${ }^{*} \mathrm{P}<0.05$, vs. rats on day 0 . Group 1, control; group 2, rotenone-treated group.

one unit of SOD activity is the amount of enzyme required to exhibit $50 \%$ dismutation of the superoxide radical.

The activity of peroxidase (POD) was determined in the supernatants of the rat brain homogenates using a colorimetric assay kit (Nanjing Jiancheng Bioengineering Institute), according to the manufacturer's instructions. In brief, the supernatants and reagents were vortexed for $10 \mathrm{sec}$ and placed in a water bath at $37^{\circ} \mathrm{C}$ for $30 \mathrm{~min}$. A color-developing agent (from the ELISA kit, RapidBio) was subsequently added and left to react for $1 \mathrm{~min}$. The absorbance at $420 \mathrm{~nm}$ was measured using an automicroplate reader.

The levels of thiols in the supernatants of the rat brain homogenates were detected using a total mercapto (-SH) measurement kit (Nanjing Jiancheng Bioengineering Institute), according to the manufacturer's instructions. In the presence of thiol compounds, colorless 5-5'-dithiobis(2-nitrobenzoic acid) (DTNB) is converted to yellow 5-mercapto-2-nitrobenzoic acid. The absorption spectrum of DTNB does not interfere with thiol detection (23). A total of $10 \mu \mathrm{l}$ of sample, standard or blank were reacted with working solutions for $5 \mathrm{~min}$ at room temperature. The optical densities of the products at $405 \mathrm{~nm}$ were detected using an automicroplate reader.

The levels of NO in the supernatants of the rat brain homogenates were determined using a commercial kit (Nanjing Jiancheng Bioengineering Institute), according to the manufacturer's instructions. $\mathrm{NO}$ is a highly reactive gas that is easily transformed into $\mathrm{NO}_{2}^{-}$and $\mathrm{NO}_{3}^{-}$in vivo, while $\mathrm{NO}_{2}^{-}$is finally transformed into $\mathrm{NO}_{3}^{-}$and $\mathrm{NO}_{3}{ }^{-}$is reduced to $\mathrm{NO}_{2}^{-}$by nitrate reductase. The concentration of $\mathrm{NO}_{2}^{-}$determined by the spectrophotometric method was used to determine the total levels of $\mathrm{NO}$ at $550 \mathrm{~nm}$.

Statistical analysis. Statistical analysis was performed using the SPSS 13.0 software (SPSS, Inc., Chicago, IL, USA). The data are presented as the mean \pm standard error of mean. The differences among the variables of the different groups were evaluated by one-way analysis of variance followed by a post hoc Student-Newmann-Keuls test. Student's t-test was used when only two sets of data were compared, while $\chi^{2}$ and Fisher's exact tests were used in the comparison of categorical data as required. $\mathrm{P}<0.05$ was considered to indicate a statistically significant difference.

\section{Results}

General results. Gastrointestinal disorders, particularly severe constipation and delayed gastric emptying, are core symptoms of PD that affect the majority of patients. Chronic rotenone exposure has been found to reproduce PD gastrointestinal neuropathology (24). The present study observed that rats in the rotenone-treated group consumed less food and water compared with rats in the control group. As shown in Fig. 1A, the body weight of rats markedly decreased following rotenone treatment for six days compared with the control group. In addition, a significant decrease was observed in the body weight of rotenone-treated rats at weeks $0,1,2$ and 3 following rotenone treatment compared with the control group rats.

During the experimental procedure, one rat succumbed in the control group at day 5 following the beginning of the experiment. By contrast, as shown in Fig 1B, six rats succumbed in the rotenone-treated group (three, one and two rats succumbed at day 7, 8 and 12, respectively, after the initiation of the rotenone treatment). No differences were observed in the survival rate among the control and rotenone-treated groups $\left(\chi^{2}=1.552\right.$; $\mathrm{P}=0.213$ ), which is partially attributable to the small sample size.

Behavioral rating scale. As shown in Fig. 2A, the behavioral scores of rotenone-treated rats were found to be significantly increased following the administration of rotenone when compared with the control rats. The rotenone-treated group scores reached a peak on day 7. The area under curve (AUC) of the behavioral rating scale of each rat was calculated. The differences between the AUCs of behavioral rating scales in rotenone-treated and control rats were analyzed statistically. As shown in Fig. 2B, a significant increase was observed in the area under the behavioral rating scale curves of the rotenone-treated rats compared with the control rats. Therefore, rotenone treatment may induce marked behavioral deficits mimicking the movement disorder of PD.

Nigral TH-positive neurons in the rat brains. PD is characterized by the loss of $\sim 50-70 \%$ of dopaminergic neurons in the substantia nigra pars compacta. TH is a rate-limiting enzyme in the catalyzed synthesis of dopamine and a transmitter and marker of dopaminergic neurons. The present study observed 
A

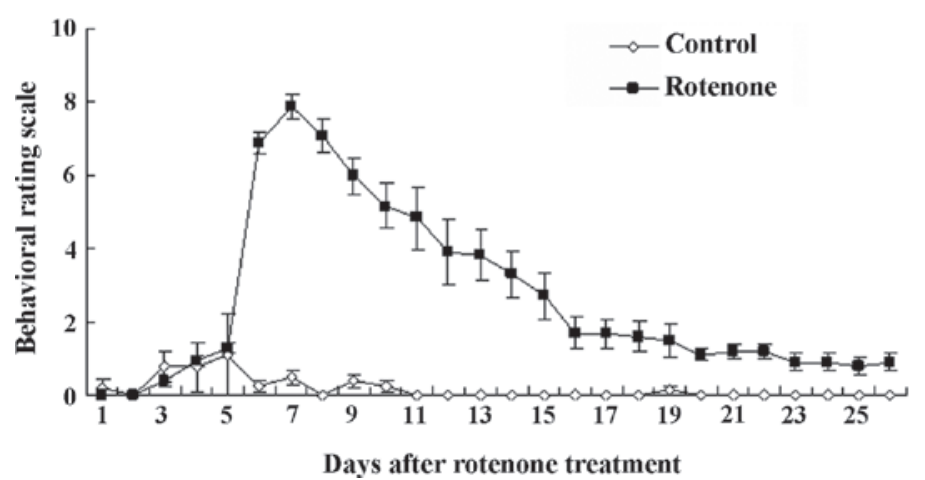

B

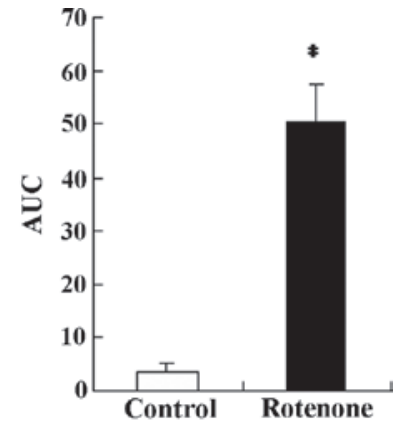

Figure 2. Effect of rotenone on the behavioral performance of the experimental rats. (A) Behavioral scores. (B) The AUC of the behavioral rating scale was calculated in each group. The data are expressed as the mean \pm standard error of the mean. " $\mathrm{P}<0.05$, vs. control group. AUC, area under the curve.

A

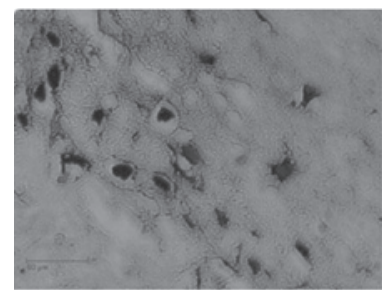

C

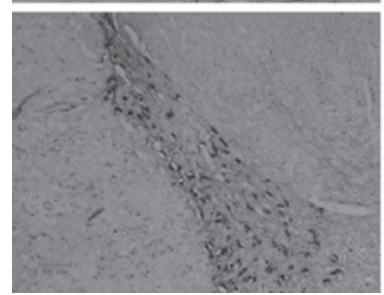

B

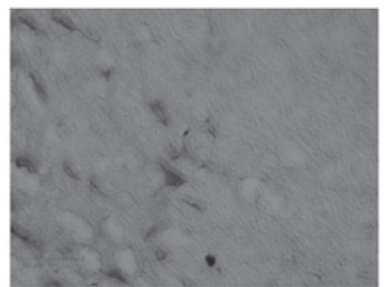

D

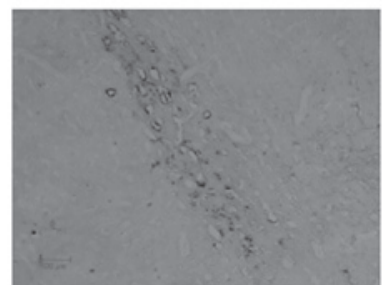

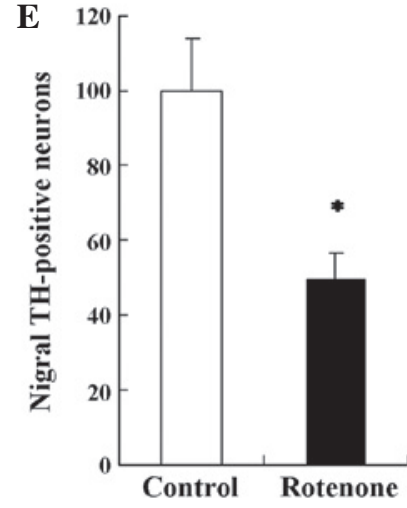

Figure 3. Effect of rotenone on the number of nigral TH-positive neurons. TH-immunoreactive staining at $\mathrm{x} 400$ magnification in the (A) control and (B) rotenone-treated rats (scale bar=50 $\mu \mathrm{m}$ ) and $\times 100$ magnification in the (C) control and (D) rotenone-treated rats (scale bar=100 $\mu \mathrm{m}$ ). (E) Relative number of nigral TH-positive neurons in each group ( $\mathrm{n}=3$ in each group). Two homologous sections from each rat with two sides of the substantia nigra were investigated at x100 magnification to determine the number of nigral TH-positive neurons. ${ }^{*} \mathrm{P}<0.05$, vs. control group. $\mathrm{TH}$, tyrosine hydroxylase.
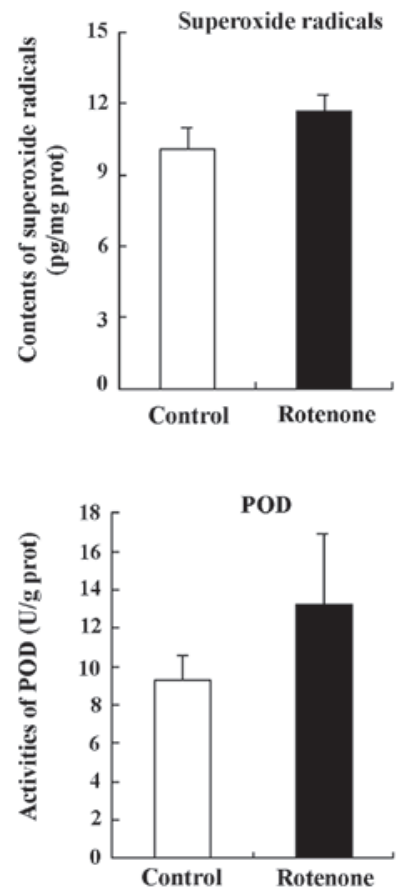
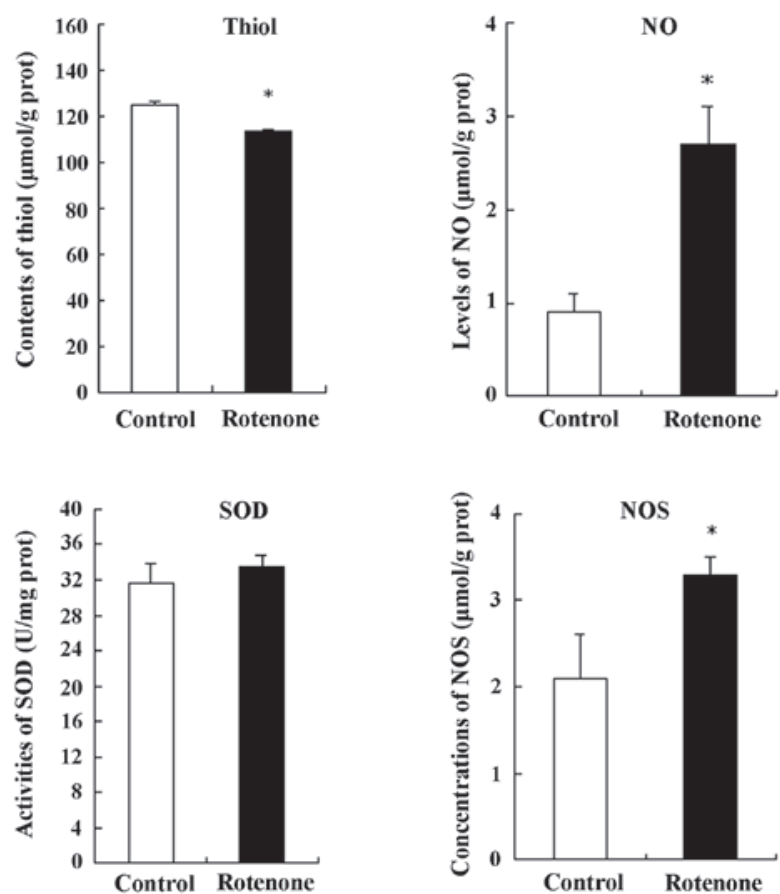

Figure 4. Effect of rotenone on oxidative, nitrosative and nitrative stress in the rat brains. Three control and six rotenone-treated rats were used to determine the levels of superoxide radicals and NO and the activities of POD, SOD and NOS, while three control and five rotenone-treated rats were used to determine the thiol levels. " $\mathrm{P}<0.05$, vs. control group. NO, nitric oxide; POD, peroxidase; SOD, superoxide dismutase; NOS, NO synthase. 
the effects of rotenone on the number of nigral TH-positive neurons in rat brains. As shown in Fig. 3, the number of nigral TH-positive neurons were found to be decreased in the rotenone-treated rats $(49.4 \pm 7.3 \%)$ when compared with the control rats $(100.0 \pm 14.2 \%)$. Therefore, the number of nigral TH-positive neurons in the rotenone-treated rats was decreased by $50.6 \%$ when compared with the control rats.

Markers of oxidative, nitrosative and nitrative stress. Previous studies have demonstrated the major contribution of reactive oxygen species, in particular NO and hydroxyl radicals, to the pathophysiology of PD (25-27). The effect of rotenone on oxidative, nitrosative and nitrative stress markers in rat brains was determined in the current study. As shown in Fig. 4, the brain thiol levels of rotenone-treated rats $(113.6 \pm 0.8 \mu \mathrm{mol} / \mathrm{g}$ protein $)$ were found to be decreased by $10.0 \%$ compared with the control rats $(125.0 \pm 1.4 \mu \mathrm{mol} / \mathrm{g}$ protein). By contrast, the brain $\mathrm{NO}$ levels of rotenone-treated rats $(2.7 \pm 0.4 \mu \mathrm{mol} / \mathrm{g}$ protein $)$ were found to be increased by $200 \%$ compared with the control rats $(0.9 \pm 0.2 \mu \mathrm{mol} / \mathrm{g}$ protein $)$. In addition, a $57.1 \%$ increase was observed in the brain NOS concentration levels of rotenone-treated rats $(3.3 \pm 0.2 \mu \mathrm{mol} / \mathrm{g}$ protein $)$ compared with the control rats $(2.1 \pm 0.5 \mu \mathrm{mol} / \mathrm{g}$ protein). No statistically significant differences were observed in the levels of superoxide radicals (10.1 \pm 0.9 vs. $11.7 \pm 0.7 \mathrm{pg} / \mathrm{mg}$ protein), POD activity (9.3 \pm 1.3 vs. $13.2 \pm 3.7 \mathrm{U} / g$ protein) and SOD activity (31.7 \pm 2.1 vs. $33.4 \pm 1.4 \mathrm{U} / \mathrm{mg}$ protein) between the control and rotenone-treated rats, respectively.

\section{Discussion}

Rotenone, a herbicide and insecticide, is the most potent member of the rotenoid family of neurotoxins that are found in tropical plants in nature. Increasing evidence has indicated that rotenone exposure is associated with an increased risk of developing PD (28). The oxidation of DA, a transmitter of dopaminergic neurons, facilitates rotenone-dependent neurotoxicity in rat substantia nigral dopaminergic neurons (29). The differences in DA concentration following intoxication, with regards to age and gender, may be due to the increased susceptibility of males and older animals to the toxic effects of neurotoxin and the aggravated process of recovery in older brains (30). Rotenone inhibits the expression of $\mathrm{TH}$, which influences the synthesis of DA in PC12 cells through the activation of plasma membrane adenosine triphosphate-sensitive potassium channels (31). However, chronic rotenone treatment has been shown to induce behavioral effects in mice with no pathological signs of Parkinsonism (32). The results of the present study revealed that $50.6 \%$ of the nigral TH-positive neurons were lost and behavioral deficits were apparent in middle-aged rats that were subacutely induced by rotenone.

Rotenone not only causes damage to nigrostriatal dopaminergic neurons, but also induces $\alpha$-synuclein aggregation and Lewy body-like formation, which are known changes in the pathology of PD $(20,33,34)$. Drolet et al demonstrated that a circumscribed exposure to environmental toxicants may lead to the delayed appearance of Parkinsonian $\alpha$-synuclein pathology in the enteric nervous system and an associated functional deficit in gastrointestinal motility (24). The present study observed a rapid weight loss in rats following rotenone administration, which may be associated with gastrointestinal dysfunction (24). Pan-Montojo et al demonstrated that rotenone may promote the release of $\alpha$-synuclein by enteric neurons (35). The released enteric $\alpha$-synuclein is then absorbed by presynaptic sympathetic neurites and retrogradely transported and accumulated at the soma. These authors hypothesized that pesticides may initiate the progression of PD pathology, which is associated with the transneuronal and retrograde axonal transport of $\alpha$-synuclein (35).

A previous study has indicated that NOS activation and peroxynitrite ion overproduction may be involved in the etiopathogenesis of PD (36). Levecque et al observed an association between PD and polymorphisms in the nNOS and iNOS genes in a community-based case-control study (37). Hancock et al showed that the interaction of NOS1 and NOS2A, the genetic risk factors for $\mathrm{PD}$, with established environmental factors (including pesticides) may modulate the effect of these factors on the pathogenesis of PD (12). However, Huerta et al found no association among PD and three polymorphisms in the eNOS, nNOS and iNOS genes (38). Furthermore, significantly low circulating levels of NO and reduced salivary NOS were demonstrated in patients with PD $(39,40)$. Chronic administration of rotenone was found to increase the NO levels in the cortex and striatum of rats, leading to significant injury in the nigrostriatal pathway $(18,19)$. The experimental results of the present study revealed a significant increase $(57.1 \%)$ in the concentration of the NOS protein and a marked increase (200.0\%) in the levels of $\mathrm{NO}$ in the brain homogenates of rats that were subacutely treated with rotenone, compared with the control rats. Overproduction of NO has been shown to result in neuronal damage and may be associated with S-nitrosylation or nitration of certain important proteins, including the S-nitrosylation of Parkin $(41,42)$, protein-disulphide isomerase (43), mitochondrial complex I (44), peroxiredoxin-2 (45) and the nitration of $\alpha$-synuclein (46) in the neurodegenerative process of PD.

A previous study has reported that the critical role of glutathione (GSH) is to maintain functional mitochondria (47). Depletion in the levels of the thiol reducing agents, GSH and GSH disulfide, is the earliest reported biochemical event occurring in the Parkinsonian substantia nigra prior to the selective loss of complex I activity associated with the disease, which is believed to contribute to subsequent dopaminergic cell death (48). The present study demonstrated a significant decrease in the levels of thiol in rotenone-induced rat brains, which is in accordance with the results observed by Di Monte et al (49).

In conclusion, the overactivation of NOS and subsequent excessive levels of $\mathrm{NO}$ in rat brains may play an important role in the behavioral deficits and loss of nigral TH-positive neurons observed in the subacute rotenone-induced rat model of PD.

\section{Acknowledgements}

This study was supported by grants from the Natural Science Foundation of Zhejiang Province (nos. Y2100035 and Y2111046), the Key Medical Discipline Construction Project of Zhejiang Province (no. GJSX-010-004), the Science and Technology Development Project of Zhejiang Province (no. 2010C33116), the Science and Technology Development Project of Shaoxing, Zhejiang Province (no. 2010A23021), the Program from Zhejiang Provincial Administration of Traditional Chinese 
Medicine (nos. 2011ZA108 and 2012ZB162) and the Program from the Department of Education of Zhejiang Province (no. Y201018615).

\section{References}

1. Gao HM and Hong JS: Gene-environment interactions: key to unraveling the mystery of Parkinson's disease. Prog Neurobiol 94: $1-19,2011$.

2. Venkateshappa C, Harish G, Mythri RB, et al: Increased oxidative damage and decreased antioxidant function in aging human substantia nigra compared to striatum: implications for Parkinson's disease. Neurochem Res 37: 358-369, 2012.

3. Fearnley JM and Lees AJ: Ageing and Parkinson's disease: substantia nigra regional selectivity. Brain 114: 2283-2301, 1991.

4. Marsden CD: Basal ganglia disease. Lancet 2: 1141-1147, 1982.

5. Collier TJ, Kanaan NM and Kordower JH: Ageing as a primary risk factor for Parkinson's disease: evidence from studies of non-human primate. Nat Rev Neurosci 12: 359-366, 2011.

6. Olanow CW, Stern MB and Sethi K: The scientific and clinical basis for the treatment of Parkinson disease. Neurology 72 (21 Suppl 4): S1-S136, 2009.

7. Coppedè F: Genetics and epigenetics of Parkinson's disease. Scientific World Journal 2012: 489830, 2012.

8. Jung J, $\mathrm{Na} \mathrm{C}$ and Huh Y: Alterations in nitric oxide synthase in the aged CNS. Oxid Med Cell Longev 2012: 718976, 2012.

9. Hwang SJ and Huh Y: Age-related changes in nitric oxide synthase in the lateral geniculate nucleus of rats. J Mol Histol 41: 129-135, 2010.

10. Gupta N, Jing Y, Collie ND, Zhang H and Liu P: Ageing alters behavioural function and brain arginine metabolism in male Sprague-Dawley rats. Neuroscience 226: 178-196, 2012.

11. Bustamante J, Czerniczyniec A, Cymeryng C and Lores-Arnaiz S: Age related changes from youth to adulthood in rat brain cortex: nitric oxide synthase and mitochondrial respiratory function. Neurochem Res 33: 1216-1223, 2008.

12. Hancock DB, Martin ER, Vance JM and Scott WK: Nitric oxide synthase genes and their interactions with environmental factors in Parkinson's disease. Neurogenetics 9: 249-262, 2008.

13. Aquilano K, Baldelli S, Rotilio G and Ciriolo MR: Role of nitric oxide synthases in Parkinson's disease: a review on the antioxidant and anti-inflammatory activity of polyphenols. Neurochem Res 33: 2416-2426, 2008.

14. Singh S, Das T, Ravindran A, et al: Involvement of nitric oxide in neurodegeneration: a study on the experimental models of Parkinson's disease. Redox Rep 10: 103-109, 2005.

15. Tsuboi Y: Environmental-genetic interactions in the pathogenesis of Parkinson's disease. Exp Neurobiol 21: 123-128, 2012.

16. van der Mark M, Vermeulen R, Nijssen PC, Mulleners WM, Sas $\mathrm{AM}$, et al: Occupational exposure to pesticides and endotoxin and Parkinson disease in the Netherlands. Occup Environ Med 71: 757-764, 2014.

17. Dhillon AS, Tarbutton GL,Levin JL, et al: Pesticide/environmental exposures and Parkinson's disease in East Texas. J Agromedicine 13: $37-48,2008$.

18. He Y, Imam SZ, Dong Z, et al: Role of nitric oxide in rotenone-induced nigro-striatal injury. J Neurochem 86: 1338-1345, 2003.

19. Bashkatova V, Alam M, Vanin A and Schmidt WJ: Chronic administration of rotenone increases levels of nitric oxide and lipid peroxidation products in rat brain. Exp Neurol 186: 235-241,2004

20. Cannon JR, Tapias V, Na HM, et al: A highly reproducible rotenone model of Parkinson's disease. Neurobiol Dis 34: 279-290, 2009.

21. Chen X, Zhao Z, Zhao Z and Mu Y: Correlation study between behavioral alteration and nigral pathological injury in rotenone-reduced rats of Parkinson's disease. Zhongguo Shen Jing Jing Shen Ji Bing Za Zhi 34: 232-234, 2008.

22. Li J, Hou Y, Zhang S, et al: Excess iron undermined bone load-bearing capacity through tumor necrosis factor- $\alpha$-dependent osteoclastic activation in mice. Biomed Rep 1: 85-88, 2013.

23. Robyt JF, Ackerman RJ and Chittenden CG: Reaction of protein disulfide groups with Ellman's reagent: a case study of the number of sulfhydryl and disulfide groups in Aspergillus oryzae -amylase, papain, and lysozyme. Arch Biochem Biophys 147: 262-269, 1971 .

24. Drolet RE, Cannon JR, Montero L and Greenamyre JT: Chronic rotenone exposure reproduces Parkinson's disease gastrointestinal neuropathology. Neurobiol Dis 36: 96-102, 2009.
25. Olanow CW and Tatton WG: Etiology and pathogenesis of Parkinson's disease. Annu Rev Neurosci 22: 123-144, 1999.

26. Beal MF: Excitotoxicity and nitric oxide in Parkinson's disease pathogenesis. Ann Neurol 44: S110-S114, 1998.

27. Jenner $P$ and Olanow CW: Oxidative stress and the pathogenesis of Parkinson's disease. Neurology 47: S161-S170, 1996.

28. Taetzsch T and Block ML: Pesticides, microglial NOX2, and Parkinson's disease. J Biochem Mol Toxicol 27: 137-149, 2013.

29. Wu YN and Johnson SW: Dopamine oxidation facilitates rotenone-dependent potentiation of N-methyl-D-aspartate currents in rat substantia nigra dopamine neurons. Neuroscience 195: 138-144, 2011.

30. Joniec I, Ciesielska A, Kurkowska-Jastrzebska I, et al: Age- and sex-differences in the nitric oxide synthase expression and dopamine concentration in the murine model of Parkinson's disease induced by 1-methyl-4-phenyl-1,2,3,6-tetrahydropyridine. Brain Res 1261: 7-19, 2009.

31. Bai Q, He J, Qiu J, et al: Rotenone induces KATP channel opening in PC12 cells in association with the expression of tyrosine hydroxylase. Oncol Rep 28: 1376-1384, 2012.

32. Richter F, Hamann M and Richter A: Chronic rotenone treatment induces behavioral effects but no pathological signs of parkinsonism in mice. J Neurosci Res 85: 681-691, 2007.

33. Coulom H and Birman S: Chronic exposure to rotenone models sporadic Parkinson's disease in Drosophila melanogaster. J Neurosci 24: 10993-10998, 2004.

34. Inden M, Kitamura Y, Takeuchi H, et al: Neurodegeneration of mouse nigrostriatal dopaminergic system induced by repeated oral administration of rotenone is prevented by 4-phenylbutyrate, a chemical chaperone. J Neurochem 101: 1491-1504, 2007.

35. Pan-Montojo F, Schwarz M, Winkler C, et al: Environmental toxins trigger $\mathrm{PD}$-like progression via increased alpha-synuclein release from enteric neurons in mice. Sci Rep 2: 898, 2012.

36. Ebadi M, Sharma SK, et al: Peroxynitrite in the pathogenesis of Parkinson's disease and the neuroprotective role of metallothioneins. Methods Enzymol 396: 276-298, 2005.

37. Levecque C, Elbaz A, Clavel J, et al: Association between Parkinson's disease and polymorphisms in the nNOS and iNOS genes in a community-based case-control study. Hum Mol Genet 12: 79-86, 2003

38. Huerta C,Sanchez-Ferrero E, Coto E, et al: No association between Parkinson's disease and three polymorphisms in the eNOS, nNOS, and iNOS genes. Neurosci Lett 413: 202-205, 2007.

39. Tuncel D, Inanc Tolun F and Toru I: Serum insulin-like growth factor- 1 and nitric oxide levels in Parkinson's disease. Mediators Inflamm 2009: 132464, 2009.

40. Huskic J, Paperniku A, Husic A, Alendar F and Mulabegovic N: Significantly reduced salivary nitric oxide synthesis in patients with Parkinson's disease. Bosn J Basic Med Sci 5: 86-89, 2005.

41. Chung KK, Dawson VL and Dawson TM: S-nitrosylation in Parkinson's disease and related neurodegenerative disorders. Methods Enzymol 396: 139-150, 2005.

42. Chung KK, Thomas B, Li X, et al: S-nitrosylation of parkin regulates ubiquitination and compromises parkin's protective function. Science 304: 1328-1331, 2004.

43. Uehara $\mathrm{T}$, Nakamura $\mathrm{T}$, Yao $\mathrm{D}$, et al: $\mathrm{S}$-nitrosylated protein-disulphide isomerase links protein misfolding to neurodegeneration. Nature 441: 513-517, 2006.

44. Borutaite V and Brown GC: S-nitrosothiol inhibition of mitochondrial complex I causes a reversible increase in mitochondrial hydrogen peroxide production. Biochim Biophys Acta 1757: 562-566, 2006 .

45. Fang J,Nakamura T, Cho DH, Gu Z and Lipton SA: S-nitrosylation of peroxiredoxin 2 promotes oxidative stress-induced neuronal cell death in Parkinson's disease. Proc Natl Acad Sci USA 104: 18742-18747, 2007.

46. Schildknecht S, Gerding HR, Karreman C, et al: Oxidative and nitrative alpha-synuclein modifications and proteostatic stress: implications for disease mechanisms and interventions in synucleinopathies. J Neurochem 125: 491-511, 2013.

47. Nunes C, Barbosa RM, Almeida L and Laranjinha J: Nitric oxide and DOPAC-induced cell death: from GSH depletion to mitochondrial energy crisis. Mol Cell Neurosci 48: 94-103, 2011.

48. Chinta SJ and Andersen JK: Reversible inhibition of mitochondrial complex I activity following chronic dopaminergic glutathione depletion in vitro: implications for Parkinson's disease. Free Radic Biol Med 41: 1442-1448, 2006.

49. Di Monte DA, Chan P and Sandy MS: Glutathione in Parkinson's disease: a link between oxidative stress and mitochondrial damage? Ann Neurol 32 Suppl: S111-S115, 1992. 